\section{Europäischer Facharzt ante portas}

\author{
Gute Nachrichten aus Cagliari: Beim Treffen der \\ Union Européenne des Médicins Spécialistes \\ (UEMS) gab es einen Durchbruch bei den Ver- \\ handlungen zum Europäischen Facharzt für \\ Allergologie und klinische Immunologie.
}

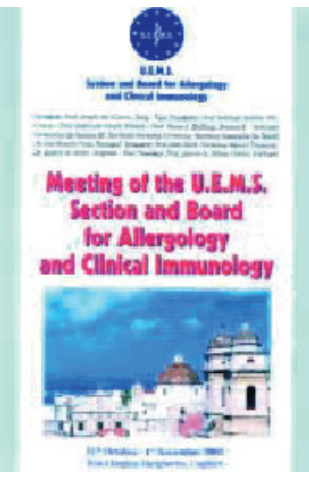

ten aus Österreich, Universitätsdozent Dr. Heinz Koffler, und des Delegierten aus Deutschland, Prof. Dr. Alexander Kapp, zu verdanken. Bisher sollte es den Internisten und Pädiatern vorbehalten sein, im so genannten „basic trunc“ die potenziellen Allergologen für 24 Monate fortzubilden. Jetzt konnte durch den Zusatz im $\$ 4$ Absatz 2 geklärt werden, dass auch andere organgebundene Fächer, wenn sie von der Ärztekammer als Weiterbildungsstätte anerkannt sind und den Inhalt des Curriculums zum Europäischen Facharzt für Allergologie und klinische Immunologie berücksichtigen, ausbilden dürfen.

In sieben europäischen Ländern ist eine Weiterbildung zum Facharzt für Allergologie und klinische Immunologie bereits möglich. Die entsprechenden Weiterbildungsinhalte sind in einem von der UEMS erarbeiteten „core curriculum" festgehalten, das auf der Website der World Allergy Organization (www.worldallergy.org) nachgelesen werden kann. Unter der Leitung von Prof. Dr. Hans-Jørgen Malling aus Kopenhagen werden zurzeit für die Auszubildenden ein so genanntes Logbuch und für den bereits praktizierenden Allergologen CME-Empfehlungen erarbeitet. Weiterbildungszentren, die sich in regelmäßigen Abständen (3-5 Jahre) von einer Kommission der UEMS überprüfen lassen, sollen ein entsprechendes Zertifikat erhalten.

Prof. Dr. Alexander Kapp, Hannover; Dr. Wolfgang Rebien, Hamburg

\title{
Atemnot während der Tage
}

\section{Frauen werden dreimal häufiger als Männer wegen eines Asthma- anfalls notfallmäßig ins Krankenhaus eingewiesen. Nahezu die Hälfte dieser Exazerbationen ereignet sich perimenstruell.}

P erimenstruelles Asthma bronchiale ist definiert als Zunahme asthmatischer Symptome und/oder Abfall der Lungenfunktionswerte kurz vor oder während der Menstruation. Die stärksten Beschwerden treten in der Regel 2-3 Tage vor Blutungsbeginn auf.

Rund $30-40 \%$ aller Asthmatikerinnen sind von perimenstruellen Exazerbationen betroffen. Diese Quote wurden mehrheitlich retrospektiv ermittelt, wobei als Symptome Atemnot, Giemen, Brustenge, Husten und Sputumvermehrung wegweisesend waren. Die Angaben der Patientinnen wurden zum Teil durch Lungenfunktionsanalysen objektiviert, wobei im Schnitt Peak-flow-Abfälle von $35-40 \%$, selten bis $80 \%$ gesehen wurden.
Alle Untersuchungen zur Pathophysiologie des perimenstruellen Asthmas kranken an der für statistische Aussagen zu kleinen Fallzahl und auch an widersprüchlichen Ergebnissen. Folgende Beobachtungen sind richtungweisend: Das Verhältnis von TH1- zu TH2-Lymphozyten verschiebt sich in der Lutealphase zugunsten von TH2, damit geht ein Anstieg von IL-4 einher. Unklar ist, ob Progesteron oder Östrogen diesen Effekt induziert. Das gleiche gilt für die erhöhte Degranulationstendenz der Mastzellen vor der Menstruation. Offen ist, ob abnorme Hormonkonzentrationen während des Zyklus eine Rolle spielen. Ein Bezug zur Dauer der Menstruation scheint nicht zu bestehen.
Diskutiert wird, ob im Rahmen der prämenstruell erhöhten Ödemneigung auch eine Schwellung der Bronchialschleimhaut mit konsekutivem Anstieg des Bronchialwiderstandes stattfindet. Bezüglich allergischer Einflüsse liegen keine sicheren Angaben vor. Das bei Menstruation oft vorkommende Unwohlsein könnte zu einer erhöhten Wahrnehmung asthmatischer Beschwerden führen und so die Grunderkrankung psychologisch modifizieren.

Diagnostisch sollte bei Verdacht auf ein perimenstruelles Asthma mindestens einmal täglich eine Peak-flowMessung vorgenommen werden. Therapeutisch muss bislang auf die konventionelle Asthmatherapie zurückgegriffen werden, zumal kontrollierte Studien über den Effekt von Hormongaben fehlen. Dies gilt auch für die hormonelle Empfängnisverhütung.

Dr. Dieter Bruchhausen, Wuppertal

\section{Literatur}

Vrieze A, Postma DS, Kerstjens HA. Perimenstrual asthma: a syndrome without known cause or cure. J Allergy Clin Immunol 2003; 112: 271-82 